\title{
Tolerance Analysis for Multilayer Optical Interconnections Integrated on a Printed Circuit Board
}

\author{
Nina Hendrickx, Student Member, IEEE, Jürgen Van Erps, Student Member, IEEE, \\ Geert Van Steenberge, Hugo Thienpont, Member, IEEE, \\ and Peter Van Daele, Member, IEEE
}

\begin{abstract}
Polymer multilayer optical interconnections have gained interest over the past few years in view of their ability to increase the integration density, increase the routing flexibility, and make full use of the characteristics of 2-D optoelectronic elements. The alignment between the functional elements in the different optical layers has to be sufficiently accurate in ensuring a high overall efficiency of the system. Numerical simulations have been used as a tool to determine whether laser ablation can be used as an alternative technology for the structuring of the functional elements of optical interconnections into a polymer optical layer. The experimentally achievable alignment accuracies are compared to tolerance ranges for an excess loss $\leq 1 \mathrm{~dB}$ obtained from the numerical study. The experimental achievements show that the alignment accuracies fall within the numerical tolerance ranges and have a good reproducibility. Experimental realizations of a two-layer multimode waveguide and inter- and out-of-planecoupling structures are discussed and shown.
\end{abstract}

Index Terms-Alignment tolerance, coupling structures, laser ablation, micromirrors, nonsequential ray tracing, optical interconnections.

\section{INTRODUCTION}

$\mathbf{T}$ HE INTRODUCTION of optical interconnections to the board level has been proposed for high-performance applications where high speed and interconnection densities are an issue. The main reason is the fact that optical interconnections offer an inherently high bandwidth and do not suffer from frequency-dependent loss and electromagnetic interference/ compatibility problems as opposed to electrical interconnections where these problems lead to a significant bandwidth limitation at high frequencies [1]. The use of a hybrid electrooptical board, which contains optical interconnections for the highspeed/bandwidth data transfer and electrical interconnects for the remaining ones, could overcome this problem [2].

Manuscript received January 15, 2007; revised May 4, 2007. This work was supported by the Flemish Institute for the Promotion of Innovation by Science and Technology (IWT) and the Flemish Fund for Scientific Research under a research fellowship to J. Van Erps. Part of this work was carried out within the framework of the Network of Excellence on Micro-Optics supported by the European Commission through the FP6 Program.

N. Hendrickx, G. Van Steenberge, and P. Van Daele are with the Department of Information Technology, Ghent University, 9000 Ghent, Belgium (e-mail: nina.hendrickx@intec.ugent.be).

J. Van Erps and H. Thienpont are with the Department of Applied Physics and Photonics, Vrije Universiteit Brussel, 1050 Brussels, Belgium.

Color versions of one or more of the figures in this paper are available online at http://ieeexplore.ieee.org.

Digital Object Identifier 10.1109/JLT.2007.901377
The integration of the optical interconnects to the board level can be achieved with the use of a polymer optical layer [3], which contains multimode optical waveguides and other passive optical elements. The material used for the optical layer and the used microstructuring technology have to be compatible with the standard printed circuit board (PCB) manufacturing and soldering processes. This requirement needs to be fulfilled in order to come to a cost-effective solution that can be adopted by the industry on a reasonable timescale [4], [5].

The interest in multilayer optical structures [6], [7], which consist of a stack of optical layers, is growing, in view of its ability to increase the integration density. In addition, the routing of the optical signals can be simplified, making full use of the 2-D characteristics of 2-D optoelectronic elements such as vertical-cavity surface-emitting laser and photodetector arrays. It is believed that two optical layers will be sufficient in fulfilling the upcoming demands concerning bandwidth and transfer speed [6]. It should however be noted that the complexity of the structure increases with each additional optical layer.

The two-layer optical structure that is being studied is briefly discussed in the next section. The alignment accuracy between the optical elements in the different layers requires special attention in view of the impact on the overall efficiency of the system. The multimode optical waveguides typically have a propagation loss around $0.1 \mathrm{~dB} / \mathrm{cm}$ and cover distances ranging from a couple of centimeters to $1 \mathrm{~m}$. In order to keep the power budget as low as possible, the efficiency of each coupling element should be within a \pm 1 -dB tolerance range with respect to the optimal value, specially when complex routing schemes are considered. We study the use of laser ablation [8] as an alternative microstructuring technology that can be used for the structuring of the functional elements of an optical interconnection into a polymer optical layer. Numerical simulations have been used as a tool to investigate whether the experimentally achievable alignment accuracies in a two-layer optical structure are within the required tolerance ranges.

\section{TWO-LAYER OPTICAL INTERCONNECTION}

Two different photopatternable materials, which are the Truemode Backplane polymer [9] and the Ormocer [10], are used for the optical layer. Both are commercially available and show excellent optical and thermal properties at the targeted 
wavelength of $850 \mathrm{~nm}$. Both materials are compatible with standard PCB manufacturing and soldering processes and have a very good adhesion to the PCB.

Each optical layer is built up as a cladding-core-cladding stack, where the cladding material has a slightly lower refractive index than the core material. This way, the light can be trapped into the core layer through total internal reflection. The optical layer contains multimode optical waveguides, which are used to guide the light in the plane of the optical layer. The waveguides have a cross section of $50 \mu \mathrm{m} \times 50 \mu \mathrm{m}$, are highly multimodal, and are mainly chosen because of the more relaxed alignment tolerances. The waveguide core features are defined into the core layer with the use of photolithography for the Ormocer and laser ablation for the Truemode material. The Ormocer waveguides are patterned into the core layer by UV exposure through a suitable mask and a subsequent development and curing step. The top cladding layer is spincoated and cured in the next step.

In the case where laser ablation is used, the material is removed on both sides of the resulting waveguide core, after which the top cladding layer is spincoated and cured [11]. Laser ablation is a flexible microstructuring technology that is fully compatible with the standard PCB manufacturing and soldering processes and is already used for the drilling of microvias into high-density electrical boards. This compatibility is one of the main reasons we want in exploring the use of laser ablation as an alternative technology for the patterning of the optical layer, which is integrated on the PCB. During the processing, the sample is placed on a computer-controlled translation stage. The length of the ablated waveguides is only limited by the maximum board size that can be handled by the translation stage.

Metallized $45^{\circ}$ micromirrors are used to deflect the light beam over $90^{\circ}$. Different technologies can be used for the fabrication of these mirrors [12], [13], but the integration into the standard PCB manufacturing processes is not always straightforward. The fabrication of the micromirror using laser ablation requires three processing steps. The $45^{\circ}$ mirror facet is ablated into the optical layer and is Au coated in the next step. The ablated cavity is finally filled with cladding material. The entire process flow is shown in Fig. 1. The core and cladding layers have a thickness of $50 \mu \mathrm{m}$; the top width of the ablated cavity is typically $100 \mu \mathrm{m}$. Some light will be reflected at the core-cladding interface prior to reaching the metallized $45^{\circ}$ facet because of the filling of the ablated trench with cladding material. Because of the tapering that occurs during the ablation, the slope of the core-cladding interface is approximately $20^{\circ}$ steeper than the $45^{\circ}$ metallized facet.

In the two-layer structure that is being studied here, both layers contain multimode optical waveguides and metallized micromirrors. The alignment between waveguides and mirrors in the top and the bottom optical layers has to be accurate enough to guarantee a high coupling efficiency. The alignment between the elements in the top and the bottom optical layers is ensured with the help of Au alignment marks. The comparison between the experimental results and the results from the numerical alignment tolerance study is given in the next section.

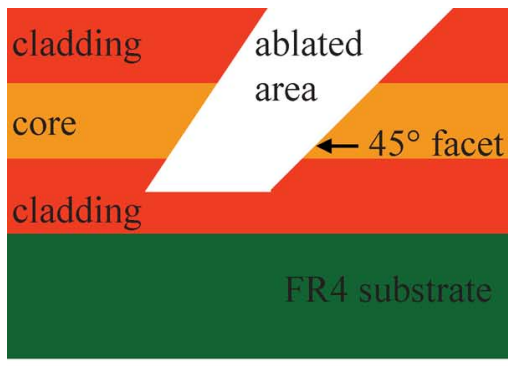

(a)

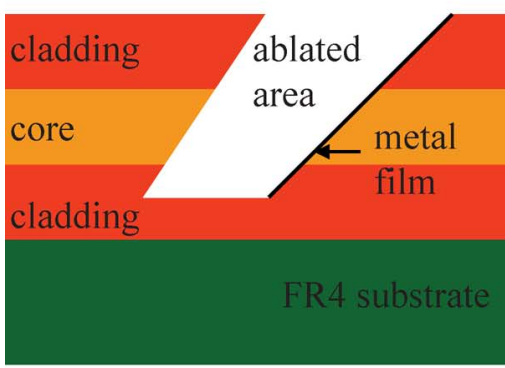

(b)

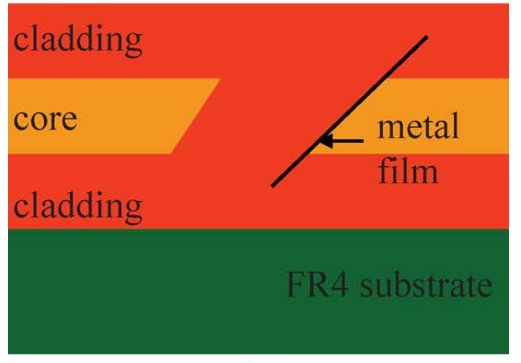

(c)

Fig. 1. Fabrication process of metallized $45^{\circ}$ micromirror. (a) Ablation $45^{\circ}$ facet. (b) Ti/Au coating of facet. (c) Filling of ablated area with cladding material.

\section{COMPARISON BETWEen NumeriCAL AND EXPERIMENTAL RESULTS}

ASAP 2005 V2R2 (Breault Research) is used to carry out the simulations. In view of the highly multimodal character of the considered structures, nonsequential ray tracing is used [5]. Fresnel losses at the different interfaces are taken into account, but the scattering losses caused by the surface roughness of the ablated structures are not included. The simulations are carried out with the technical parameters given in Table I. Light with a wavelength of $850 \mathrm{~nm}$ is coupled into the polymer multimode waveguide, which is either patterned into the Truemode or Ormocer optical layer. The numerical aperture (NA) of multimode waveguides is 0.3 for the Truemode and 0.2 for the Ormocer material. The waveguides have a cross section of $50 \mu \mathrm{m} \times 50 \mu \mathrm{m}$ and a length of $10 \mathrm{~cm}$, in order to obtain a sufficient degree of mode scrambling. The distance between the input fiber and the input facet and the output facet and the optical receiver is $10 \mu \mathrm{m}$.

\section{A. Ablated Waveguides and Micromirrors}

$\mathrm{KrF}$ excimer laser $(248 \mathrm{~nm})$ is used for the ablation of the Truemode multimode waveguides and the $45^{\circ}$ facets of the micromirrors. Polymer materials typically show a high 
TABLE I

TeChNicAl Parameters USED For THe Simulations

\begin{tabular}{|c|c|}
\hline Input fiber & $\begin{array}{c}\text { MMF diameter } 50 \mu \mathrm{m}, \\
\mathrm{NA}=0.2\end{array}$ \\
\hline Output fiber & $\begin{array}{c}\text { MMF diameter } 100 \mu \mathrm{m}, \\
\mathrm{NA}=0.29\end{array}$ \\
\hline Flat detector & $100 \mu \mathrm{m}$ square \\
\hline Refractive index Truemode $^{T M}$ core & 1.5563 \\
\hline Refractive index Truemode $^{T M}$ cladding & 1.5266 \\
\hline Refractive index Ormocer $囚$ core & 1.55475 \\
\hline Refractive index Ormocer $囚$ cladding & 1.5306 \\
\hline
\end{tabular}

$\mathrm{I}=125 \mathrm{um} \quad \mathrm{l}=65 \mathrm{um} \quad \mathrm{I}=55 \mathrm{um}$

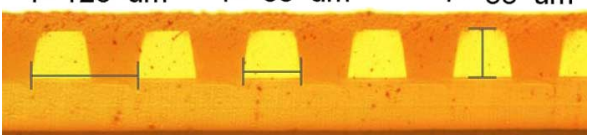

Fig. 2. Cross section of an array of laser ablated waveguides in Truemode.

absorption in the UV region, which allows for a clean ablation with low deposition of debris. During the processing, the sample is placed on a computer-controlled translation stage, which has an accuracy of $1 \mu \mathrm{m}$. The laser beam can be tilted with respect to the sample, which eases the structuring of angled features considerably. The sample can thus stay horizontally on the translation stage during the ablation of the mirrors.

There is always a certain degree of tapering during the ablation. This tapering is responsible for the slightly trapezoidal cross section of the ablated waveguides, as shown in Fig. 2. Simulations show that this trapezoidal form has a very limited influence on the performance of the waveguide in case the angle between the actual sidewall and the straight sidewall is smaller than $25^{\circ}$, as is the case for the ablated waveguides. The rootmean-square (rms) surface roughness of the waveguide side walls has been measured with a noncontact optical profiler (Wyko NT3000) and has an average value of $35 \mathrm{~nm}$ on a scan area of $46 \mu \mathrm{m} \times 109 \mu \mathrm{m}$. The waveguides have a propagation loss of $0.12 \mathrm{~dB} / \mathrm{cm}$ at $850 \mathrm{~nm}$ [11].

The $\mathrm{KrF}$ excimer laser beam is tilted under a suitable angle for the ablation of the $45^{\circ}$ facet, taking into account the tapering angle. The Au coating is evaporated only onto the $45^{\circ}$ facet by covering the sample with a suitable mask and placing it under a $45^{\circ}$ angle in the vacuum chamber. The $45^{\circ}$ facet has an average rms surface roughness of $60 \mathrm{~nm}$ for the Truemode and $70 \mathrm{~nm}$ for the Ormocer material, measured over a scan area of $46 \mu \mathrm{m} \times 109 \mu \mathrm{m}$. The angle accuracy of the facet is determined by the tilt angle of the laser beam and has been controlled with a contact stylus profiler (Dektak) [14]. The angle of the facet is highly reproducible and has an average deviation of $\pm 1^{\circ}$. Simulations on the influence of the facet angle on the coupling efficiency show us that an angle deviation of $\pm 2^{\circ}$ has a neglectable influence on the performance of the mirror, mainly because of the presence of the highly reflective Au coating. The cross section of a metallized $45^{\circ}$ micromirror is given in Fig. 3 . The top width of the filled cavity is typically $100 \mu \mathrm{m}$; the angle of the core-cladding interface is typically $65^{\circ}$ with respect to the plane of the optical waveguides.

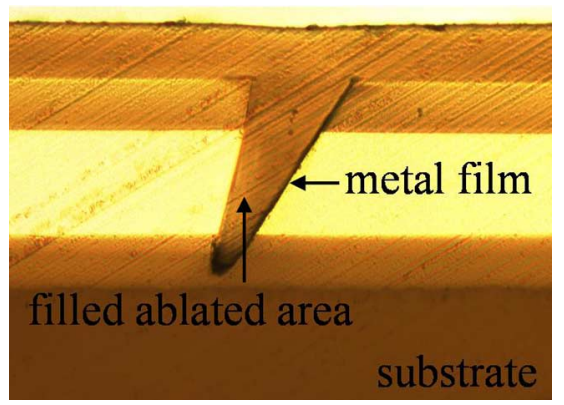

Fig. 3. Cross section of a metallized $45^{\circ}$ micromirror in Truemode.

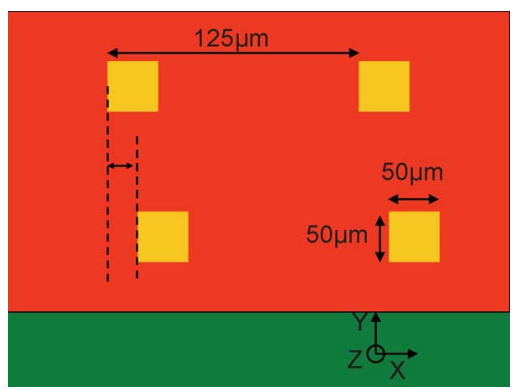

Fig. 4. Misalignment along the $x$-axis of the bottom waveguide with respect to the top waveguide in a two-layer waveguide structure.

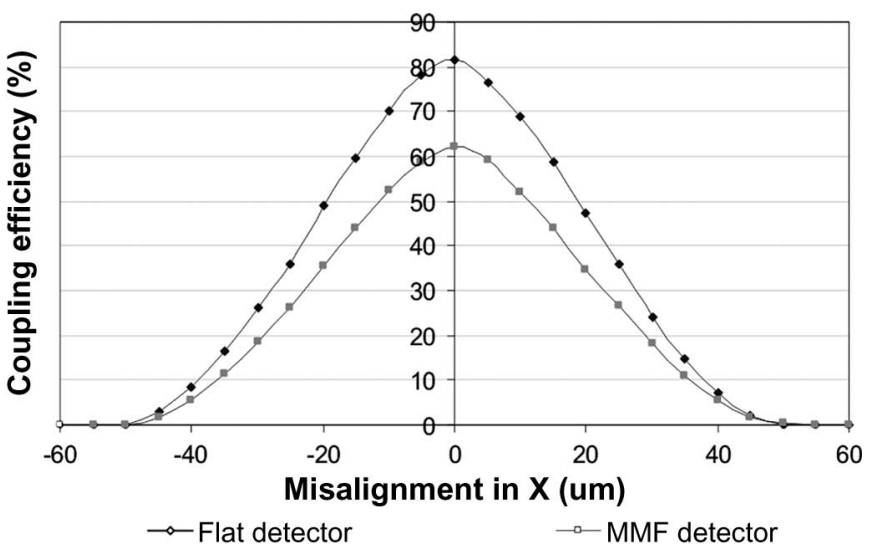

Fig. 5. Alignment tolerance along the $x$-axis for the bottom waveguide in a two-layer waveguide structure in Truemode material for the case of a flat and Multimode Optical Fiber (MMF) detector.

\section{B. Two-Layer Waveguide Structure}

The tolerance for a misalignment of the bottom waveguide in a two-layer waveguide structure has been simulated by shifting the bottom waveguide along the $x$-axis. The light signal is coupled out of the plane of the optical layer using a metallized micromirror and is then detected by either a flat isotropic detector or a multimode optical fiber (MMF) detector. Fig. 4 schematically shows the cross section of a two-layer waveguide structure where the bottom waveguide is misaligned with respect to the top waveguide. The cladding layer between the two waveguide cores has a thickness of $100 \mu \mathrm{m}$. The propagation direction through the waveguides is along the $z$-axis, perpendicular to the $x y$-plane as defined in the figure. The alignment tolerance curves are given in Fig. 5. From these curves, we can conclude that the alignment tolerance range for 


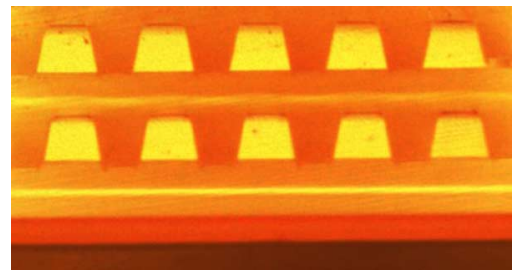

Fig. 6. Two-layer optical waveguide structure ablated in Truemode material. The waveguides have a cross section of $50 \mu \mathrm{m} \times 50 \mu \mathrm{m}$ and are positioned on a pitch of $125 \mu \mathrm{m}$.

an excess loss $\leq 1 \mathrm{~dB}$ is [ $-10 \mu \mathrm{m}, 10 \mu \mathrm{m}]$ for both detector and material types.

For the experimental realization, the alignment between the waveguides is done with the help of the Au alignment marks, which are evaporated on top of a planarization layer on the FR4 substrate. The planarization layer is used in compensating for the deformations of the FR4 substrate. The camera of the ablation setup is tilted with respect to the surface of the sample, causing a certain horizontal shift over the sample surface when the focus is moved from the alignment crosses, which are marked on a lower lying layer, to the top layer, which is going to be structured. The shift is however limited to one direction, so by ablating an alignment pulse on the top layer at a predefined distance from the alignment cross and then rotating the substrate over $90^{\circ}$, the shift over the sample surface can be compensated.

The cross section of a two-layer waveguide structure ablated in Truemode is given in Fig. 6. The alignment accuracy is $\leq 5 \mu \mathrm{m}$, which is measured by SEM and optical microscopy. Multiple experiments have been carried out and confirm the good reproducibility. In the case where Ormocer materials are used, the definition of the waveguides is done photolithographically. The alignment accuracy is then determined by the alignment accuracy of the mask-aligner setup that is being used, which is typically 5 to $10 \mu \mathrm{m}$.

\section{Out-of-Plane Turning Mirrors}

The studied structure is shown in Fig. 7. The pitch between the outcoupled spots is $250 \mu \mathrm{m}$, in accordance with the pitch of the optical source or detector array. The light is coupled out of the plane of the optical layer using metallized $45^{\circ} \mathrm{mi}-$ cromirrors toward a detector array. The size of the flat detector is chosen in accordance with components available at our lab. In this way, we can do experiments in the future and compare them to the simulation results.

The alignment tolerance curve along the $z$-axis for both top and bottom mirrors has been simulated by shifting the concerned mirror around its ideal position, where there is no misalignment. The alignment tolerance range gives the acceptable misalignment for an excess loss $\leq 1 \mathrm{~dB}$. The alignment tolerance curve for a misalignment along the $z$-axis of the bottom and the top mirrors in Truemode for the case of a flat detector is given in Fig. 8. The curves are not symmetrical around the theoretically ideal position. This is caused by the buildup of the micromirror, where the light beam has to pass the tilted core-cladding interface prior to reaching the metallized facet.

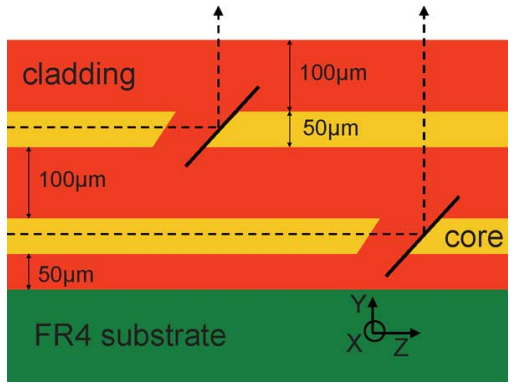

Fig. 7. Two-layer out-of-plane turning structure.

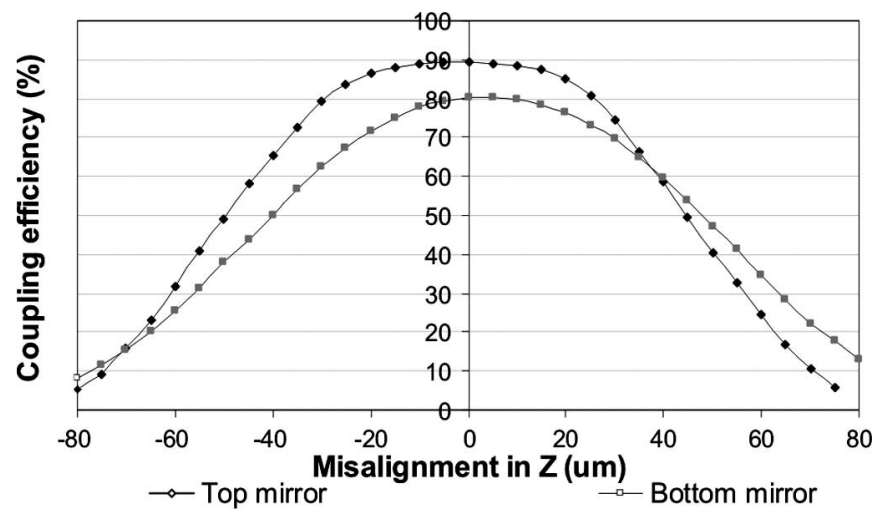

Fig. 8. Alignment tolerance curve of the top and the bottom mirrors in Truemode for a misalignment along $z$ for the case of the flat detector.

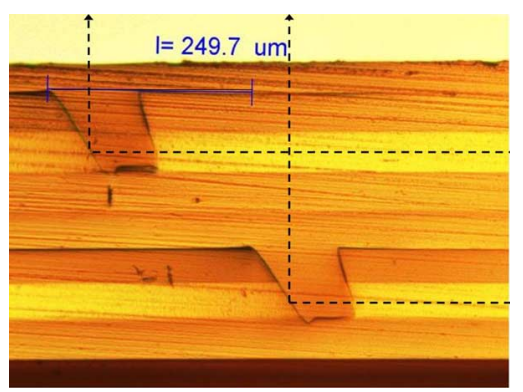

Fig. 9. Cross section of a two-layer out-of-plane turning-mirror structure in Ormocer. The darker color in the picture is caused by the manual polishing process used to make the cross section.

The spot coupled out at the bottom mirror, however, has spread over a larger distance than the top mirror; the tolerance ranges are therefore not identical. The alignment tolerances ranges are $[-30 \mu \mathrm{m}, 35 \mu \mathrm{m}]$ for the bottom and $[-35 \mu \mathrm{m}, 30 \mu \mathrm{m}]$ for the top mirror. The tolerance ranges are identical for both studied materials.

The optical power that is captured by the MMF detector is determined by its NA, and for this reason, the maximum coupling efficiency is lower than for the flat detector. The simulated tolerance range is $[-40 \mu \mathrm{m}, 40 \mu \mathrm{m}]$ for the bottom and $[-40 \mu \mathrm{m}, 35 \mu \mathrm{m}]$ for the top mirror. The apparently more relaxed tolerance range is due to the step-index definition of the MMF detector leading to the existence of rays propagating in the fiber cladding.

Fig. 9 shows the cross section of a two-layer out-of-plane turning-mirror structure ablated in Ormocer, where the outcoupled spots are separated by $250 \mu \mathrm{m}$. The core layers have a 
thickness of $50 \mu \mathrm{m}$; the cladding between the two core layers has a thickness of $100 \mu \mathrm{m}$. The $45^{\circ}$ facets are in this case not metallized, because this requires extra processing steps, which are not necessary to check the achievable alignment accuracy. The alignment procedure used here is similar to the one used for the two-layer waveguide structure. The alignment accuracy of the pitch between the top and the bottom mirrors is $\leq 5 \mu \mathrm{m}$. Multiple experiments have been carried out in both polymer materials and confirmed the good reproducibility of the results. Taking into account the tolerance range obtained from the numerical study, we can conclude that we can use laser ablation for the structuring of the $45^{\circ}$ facets into the twolayer optical waveguide structure. Loss measurements will be carried out in the near future in comparing the experimentally obtained coupling efficiency and the theoretical value. If necessary, microlenses can be ablated into the top cladding layer to increase the coupling efficiency between the mirror and the detector [15].

The tolerance for a mechanical misalignment of the detector along the $x$-axis has been studied by shifting the detector with respect to its ideal position. The alignment tolerance curves for the top and the bottom mirrors are symmetrical around the ideal position for both detector types, which is logical, since we are moving along the metallized facet. The alignment tolerance range for an excess loss $\leq 1 \mathrm{~dB}$ is $[-30 \mu \mathrm{m}, 30 \mu \mathrm{m}]$ with respect to the bottom and the top mirrors for the flat detector for both studied materials. In case the MMF detector is used, the tolerance ranges are $[-20 \mu \mathrm{m}, 20 \mu \mathrm{m}]$ with respect to the bottom and $[-40 \mu \mathrm{m}, 40 \mu \mathrm{m}]$ with respect to the top mirror.

The detector can be placed with a standard flip-chip technology onto the contacts with a precision of $\pm 5 \mu \mathrm{m}$. In case the contacts are defined photolithographically with respect to the mirrors, the achievable overall precision is $\pm 10 \mu \mathrm{m}$ [16].

\section{Interplane Turning Mirrors}

The metallized $45^{\circ}$ micromirrors can also be used in coupling light between waveguides in the top and the bottom optical layers. Two possible configurations, which are shown Fig. 10, have been studied. In the first configuration, the light undergoes a double $90^{\circ}$ bend with conservation of the propagation direction; in the second one, there is also a change in the propagation direction. As shown in Fig. 10, the reflection at the top mirror occurs directly at the metallized facet without crossing through the cladding-filled cavity.

The alignment tolerance curve for the first configuration for a misalignment along the $z$-axis of the top mirror with respect to the bottom mirror is symmetrical around the ideal position $z=0$. The outcoupled signal is captured by the detector, which is placed at the output facet of the waveguides. The tolerance range for an excess loss $\leq 1 \mathrm{~dB}$ is $[-10 \mu \mathrm{m}, 10 \mu \mathrm{m}]$ for both detector types and studied materials.

Fig. 11 shows the cross section of the experimental realization of an interplane turning-mirror configuration, where the light undergoes a double $90^{\circ}$ bend with conservation of the propagation direction. The core layers have a thickness of $50 \mu \mathrm{m}$, the thickness of the cladding layer between the two core layers is $100 \mu \mathrm{m}$. As before, the alignment is assured with

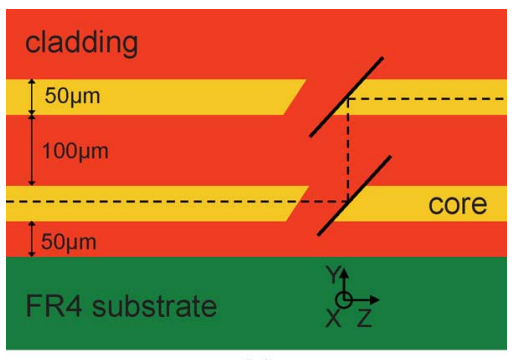

(a)

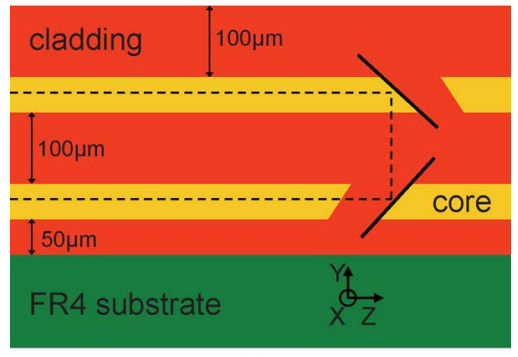

(b)

Fig. 10. Interplane turning mirrors. (a) Double $90^{\circ}$. (b) Double $90^{\circ}$ bend with change of direction.

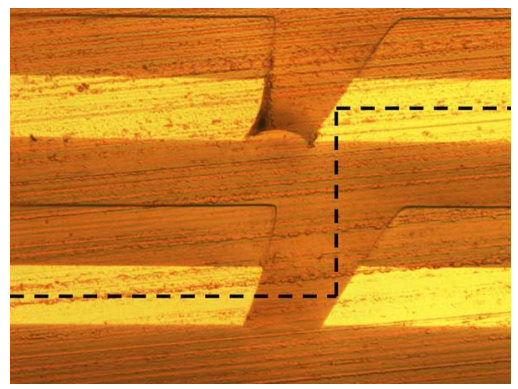

Fig. 11. Cross section of an interplane turning-mirror configuration in Truemode material where the light signal undergoes a double $90^{\circ}$ bend with conservation of the propagation direction.

the use of the $\mathrm{Au}$ alignment marks and the use of an extra alignment pulse on the top layer at a predefined distance from the alignment crosses. The achievable alignment accuracy is $\leq 5 \mu \mathrm{m}$, in accordance with the results obtained for the twolayer waveguide and out-of-plane turning-mirror configuration.

The alignment tolerance curve for a misalignment in $z$-direction of the top mirror in the double $90^{\circ}$ bend, which is shown in Fig. 10(b), where the light is coupled from one layer to the other, and the propagation direction is changed, as shown in Fig. 12. The maximum coupling efficiency is not achieved for $z=0$ but for $z=-5 \mu \mathrm{m}$. This is again caused by the buildup of the metallized micromirror. The alignment tolerances for an excess loss $\leq 1 \mathrm{~dB}$ are $[-30 \mu \mathrm{m}, 20 \mu \mathrm{m}]$ for the flat detector and $[-20 \mu \mathrm{m}, 10 \mu \mathrm{m}]$ for the MMF detector.

Fig. 13 shows the cross section of an experimental realization of an interplane turning-mirror configuration, where the light undergoes a double $90^{\circ}$ bend with change of the propagation direction. The alignment between the mirrors in the top and the bottom layers is in this case not as straightforward as for the other configuration. The $45^{\circ}$ facet of the micromirror is ablated with the tilted $\mathrm{KrF}$ excimer laser beam. During the ablation of the mirrors, the alignment cross of the camera is placed on the 


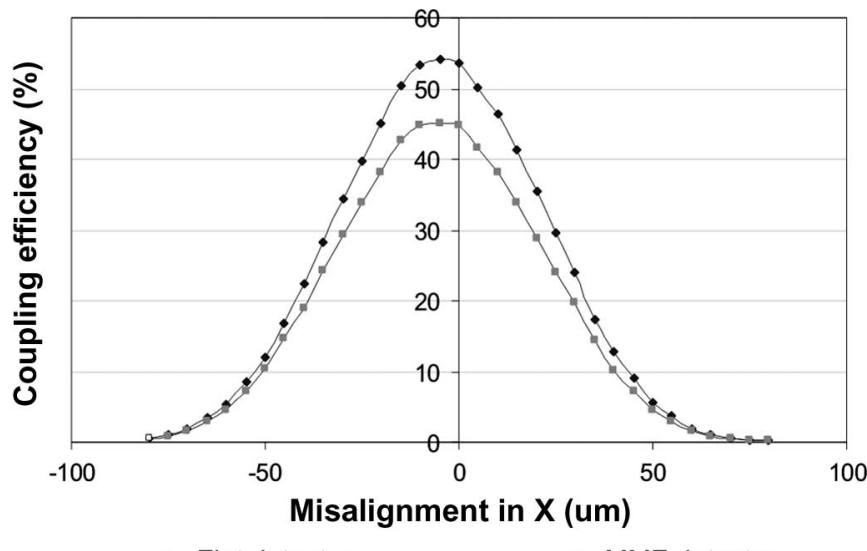

$\rightarrow$ Flat detector

$\rightarrow-$ MMF detector

Fig. 12. Alignment tolerance curve for an interplane coupling configuration in Truemode with change of propagation direction.

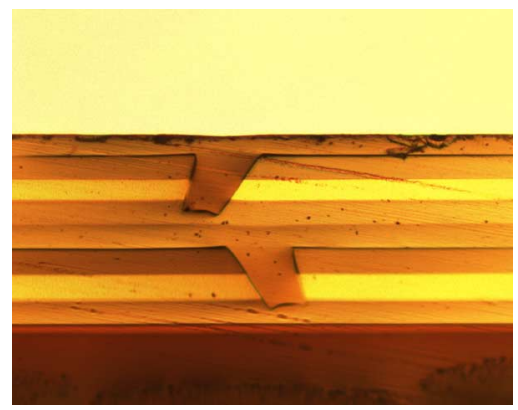

Fig. 13. Cross section of an interplane turning-mirror configuration in Ormocer material where the light signal undergoes a double $90^{\circ}$ bend with change of the propagation direction.

top layer at the edge of the steeper interface and not at the edge of the $45^{\circ}$, because this edge is much more clearly visible. The sample has to be rotated over $180^{\circ}$ to do the ablation of the top mirror. The shift that is required in obtaining a good alignment between both mirrors is determined by both the thickness of the cladding layer on top of the core layer and the width of the ablated cavity. Spincoating is used for the application of the layers; the achievable accuracy on the layer thickness depends on the spin speed that is used. For Ormocer, the cladding material is spinned at a speed of $1200 \mathrm{r} / \mathrm{min}$, which gives a layer thickness of $50 \mu \mathrm{m} \pm 2 \mu \mathrm{m}$. Truemode has a much lower viscosity and is consequently spinned at a lower speed; spinning at a speed of $250 \mathrm{r} / \mathrm{min}$ gives a cladding layer with a thickness of $50 \mu \mathrm{m} \pm 5 \mu \mathrm{m}$. The width of the ablated cavity depends on the pulse energy of the laser and the number of pulses that are used. The pulse energy of the excimer laser is monitored with an energy meter prior to the ablation of the mirrors and can be adapted to the desired value with the use of an attenuator. Multiple experiments have been carried out, and these show us that the achievable alignment accuracy is $\leq 10 \mu \mathrm{m}$. This is not as accurate as for the other studied configurations but lies within the tolerance range resulting from the simulations.

\section{CONCLUSION}

A two-layer optical structure that contains multimode waveguides and $45^{\circ}$ micromirrors, the basic building blocks of an optical interconnection, has been studied both numerically and experimentally. Laser ablation has been used for the microstructuring of the waveguides and the mirrors into the polymer optical layer. Both laser ablation and the studied polymer materials, which are the Truemode Backplane polymer and the Ormocer, are compatible with the standard PCB manufacturing and soldering processes. The experimental results show us that the achievable alignment accuracy in a two-layer mirror and waveguide structure is $\leq 5 \mu \mathrm{m}$. The alignment tolerances for the two-layer multimode waveguide and out-ofplane and interplane turning-mirror configurations have been analyzed numerically with a nonsequential ray-tracing program. The alignment tolerances giving an excess loss $\leq 1 \mathrm{~dB}$ on the overall coupling efficiency of the simulated systems have been drawn from the obtained alignment tolerance curves. The experimentally achievable alignment accuracy lies well within the alignment tolerance ranges required for an excess loss $\leq 1 \mathrm{~dB}$. Future work consists of coupling loss measurements on the two-layer out-of-plane and interplane turning-mirror configurations and the study of the influence of a misalignment on the efficiency of the system. The influence of the scattering losses caused by the surface roughness of the ablated areas will also be taken into account in future simulations.

\section{REFERENCES}

[1] D. Miller and H. Ozaktas, "Limit to the bit-rate capacity of electrical interconnects from the aspect ratio of the system architecture," J. Parallel Distrib. Comput., vol. 41, no. 1, pp. 42-52, Feb. 1997.

[2] E.-H. Lee, S. G. Lee, B. H. O, S. G. Park, and K. H. Kim, "Fabrication of a hybrid electrical-optical printed circuit board (EOPCB) by lamination of an optical printed circuit board (O-PCB) and an electrical printed circuit board (E-PCB)," in Proc. Photon. West, vol. 6126, p. 61 260L-1.

[3] L. Eldada and L. W. Shacklette, "Advances in polymer integrated optics," IEEE J. Sel. Topics Quantum Electron., vol. 6, no. 1, pp. 54-68, Jan./Feb. 2000.

[4] C. Berger, B. J. Offrein, and M. Schmatz, "Challenges for the introduction of board-level optical interconnect technology into product development roadmaps," Proc. SPIE, vol. 6124, p. 61 240J-1, 2006.

[5] E. Griese, "Optical interconnection technology for PCB applications," PC Fab, vol. 25, no. 6, pp. 20-36, 2002.

[6] A. Glebov, B. G. Lee, S. Aoki, D. Kudzuma, J. Roman, M. Peters, L. Huang, D. S. Zhou, and K. Yokouchi, "Integrated waveguide microoptic elements for 3D routing in board-level optical interconnects," Proc. SPIE, vol. 6126, p. $61260 \mathrm{~N}-1,2006$.

[7] J.-S. Kim and J.-J. Kim, "Stacked polymeric multimode waveguide arrays for two-dimensional optical interconnects," J. Lightw. Technol., vol. 22, no. 3, pp. 840-845, Mar. 2004.

[8] G. Van Steenberge, P. Geerinck, S. Van Put, J. Van Koetsem, H. Ottevaere, D. Morlion, H. Thienpont, and P. Van Daele, "MT-compatible laserablated interconnections for optical printed circuit boards," J. Lightw. Technol., vol. 22, no. 9, pp. 2083-2090, Sep. 2004.

[9] F. Tooley et al., "Optically written polymers used as optical interconnects and for hybridisation," Opt. Mater., vol. 17, no. 1, pp. 235-241, Jun. 2001.

[10] U. Streppel, P. Dannberg, C. Wächter, A. Bräuer, L. Fröhlich, R. Houbertz, and M. Popall, "New wafer-scale fabrication method for stacked optical waveguide interconnects and 3D micro-optic structures using photoresponsive (inorganic-organic hybrid) polymers," Opt. Mater., vol. 21, no. 1, pp. 475-483, Jan. 2002.

[11] G. Van Steenberge, N. Hendrickx, E. Bosman, J. Van Erps, H. Thienpont, and P. Van Daele, "Laser ablation of parallel optical interconnect waveguides," IEEE Photon. Technol. Lett., vol. 18, no. 9, pp. 1106-1108, May 2006.

[12] A. Glebov, J. Roman, M. G. Lee, and K. Yokouchi, "Optical interconnect modules with fully integrated reflector mirrors," IEEE Photon. Technol. Lett., vol. 17, no. 7, pp. 1540-1542, Jul. 2005.

[13] Y. Liu, L. Lin, C. Choi, B. Bihari, and R. T. Chen, "Optoelectronic integration of polymer waveguide array and metal-semiconductor-metal photodetector through micromirror couplers," IEEE Photon. Technol. Lett., vol. 13, no. 4, pp. 355-357, Apr. 2001. 
[14] N. Hendrickx, G. Van Steenberge, P. Geerinck, J. Van Erps, H. Thienpont, and P. Van Daele, "Laser ablated coupling structures for stacked optical interconnections on printed circuit boards," in Proc. SPIE Photon. Eur., 2006, p. 618503.

[15] K. Naessens, H. Ottervaere, P. Van Daele, and R. Baets, Appl. Surf. Sci., vol. 208, p. 159, 2002.

[16] J. van der Linden, P. De Dobbelaere, P. Van Daele, and M. Diemeer, "High-density and alignment-tolerant integration of monitoring photodetector arrays onto polymeric guided-wave components," IEEE Trans. Adv. Packag., vol. 22, no. 4, pp. 534-540, Nov. 1999.

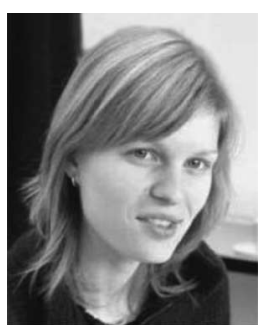

Nina Hendrickx (S'04) was born in Antwerp, Belgium, in 1981. She received the degree in electrical engineering from Ghent University, Ghent, Belgium, in 2004. She is currently working toward the Ph.D. degree in electrical engineering in the Department of Information Technology, Ghent University, with financial support from the Institute for the Promotion of Innovation by Science and Technology (IWT), Flanders, Belgium.

She is the author or coauthor of two SCI-stated journal papers and more than 10 publications in international conference proceedings. Her research interests include optical interconnections, microstructuring technologies, and coupling structures.

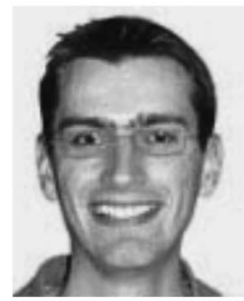

Jürgen Van Erps (S'03) was born in Etterbeek, Belgium, in 1980. He received the degree in electrotechnical engineering, major in photonics, from the Vrije Universiteit Brussel (VUB), Brussels, Belgium, in 2003. He is currently working toward the Ph.D. degree in the Department of Applied Physics and Photonics, VUB, under a fellowship from the Flemish Fund for Scientific Research (FWO-Vlaanderen).

$\mathrm{He}$ is the author of eight SCI-rated journal papers. Mr. Van Erps is a member of the International Society for Optical Engineers (SPIE), the Optical Society of America, and the IEEE Lasers and Electro-Optics Society. He was an Invited Speaker in four international conferences.

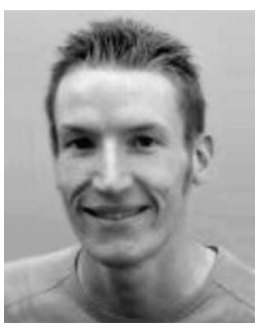

Geert Van Steenberge was born in Zottegem, Belgium, in 1979. He received the degree in electrical engineering and the Ph.D. degree in engineering sciences from Ghent University, Ghent, Belgium, in 2002 and 2006, respectively.

$\mathrm{He}$ is currently with the Thin-Film Components Group (TFCG) Microsystems Group, Ghent University, supervising work on optical interconnects. He is the author or coauthor of five SCI-stated journal papers and more than 30 publications in international conference proceedings. His current research interests include material microstructuring by using laser ablation, the integration of optical interconnections on both rigid and flexible circuit boards, and optoelectronics in general.

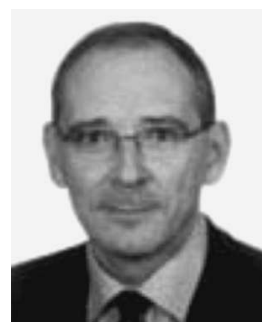

Hugo Thienpont (M'99) was born in Ninove, Belgium, in 1961. He received the degree in electrotechnical engineering and the Ph.D. degree in applied sciences from the Vrije Universiteit Brussel (VUB), Brussels, Belgium, in 1984 and 1990, respectively.

He was a Professor of photonics with the Faculty of Applied Sciences, VUB, in 1994. He was with the Department of Applied Physics and Photonics, VUB, as the Research Director in 2000, where he has been the Chair since 2004. He is currently the Coordinator of several basic research and networking projects such as the European Network of Excellence on Micro-Optics (NEMO). In addition to academic-oriented research projects, he manages microphotonics-related industrial projects with companies like Barco, Agfa-Gevaert, Tyco, and Umicore. He was a Guest Editor of several special issues on optical interconnects, including Applied Optics, and is the Editor of 15 conference proceedings. He is a coholder of ten patents. He is the author of $80 \mathrm{SCI}$-stated journal papers, more than 250 publications in international conference proceedings, and five book chapters.

Dr. Thienpont is a Fellow of the International Society for Optical Engineers (SPIE) and a member of the Electrical Optical Systems Inc., the IEEE Laser and Electro-Optics Society, and the Optical Society of America. He received the International Commission for Optics Prize and the Ernst Abbe Medal from Carl Zeiss in 1999, the IEEE LEOS Distinguished Lecturer Award in 2003, and the SPIE President's Award for his dedicated service to the European Community in 2005. He was a Guest Editor of the IEEE Journal OF SELECTED Topics on Quantum Electronics. He was an Invited Speaker in 40 international conferences. He is the General Chair of the SPIE Photonics Europe Conference.

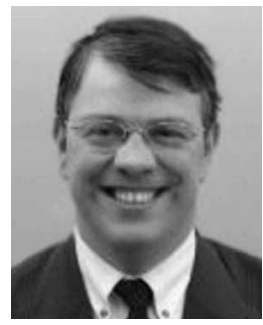

Peter Van Daele (M'91) received the Ph.D. degree in electrical engineering from Ghent University, Ghent, Belgium, in 1988.

He was a member of IMEC Staff with the Department of Information Technology, Ghent University, responsible for research on the processing of III-V optoelectronic devices. He was a part-time Professor in 1993. He is currently with the Department of Information Technology, Ghent University. Since 2001, his work has involved optical packaging and optical interconnections, with emphasis on coupling to fiber arrays, integration on printed circuit boards, and use of laser processing techniques. He is the author or coauthor of about 200 publications in optoelectronic components and technology. 